\title{
Transmission parameters of an anisotropic layered structure in the waveguide
}

\section{Andrzej Dukata, Waldemar Susek}

Andrzej Dukata, Waldemar Susek, "Transmission parameters of an anisotropic layered structure in the waveguide," Proc. SPIE 11442,

Radioelectronic Systems Conference 2019, 114420A (11 February 2020); doi: $10.1117 / 12.2565584$

SPIE. Event: Radioelectronic Systems Conference 2019, 2019, Jachranka, Poland 


\title{
Transmission parameters of an anisotropic layered structure in the waveguide
}

\author{
Andrzej Dukata*, Waldemar Susek \\ Military University of Technology, Electronic Department, 00-908 Warsaw 49, 2 Kaliski St., Poland
}

\begin{abstract}
The solution of the boundary value problem of electromagnetic wave transmission through an anisotropic layered structure placed in a rectangular waveguide is presented. The main axes of the crystallographic system of the layers are oriented parallel to the edges of the waveguide. For transmission through a single slab, the resulting scattering matrix elements $S_{11}$ and $S_{21}$ are transformed to the form known from the Nicolson-Ross-Weir model. This gives the possibility of using this model to retrieve complex material parameters (components of the permittivity and permeability tensors).
\end{abstract}

Keywords: permittivity tensor, permeability tensor, Nicolson-Ross-Weir model, scattering matrix elements $S_{11}$ and $S_{21}$

\section{INTRODUCTION}

Recent trends in left-handed metamaterials (LHM) have led to a renewed interest on retrieving their effective permittivity $\varepsilon$ and permeability $\mu^{1-4}$. Much of the LHMs realized so far are assumed to be biaxially anisotropic. Other important classes of man-made materials such as carbon-fiber reinforced plastics (CFRP) are characterized by uniaxial anisotropy ${ }^{5}$.

One of the most important methods for determining complex material parameters is the Nicolson-Ross-Weir (NRW) method. In 1970, Nicolson and Ross ${ }^{6}$ gave the formulas for extraction of the complex permittivity and the permeability of an isotropic sample from measurements of the scattering matrix elements $S_{11}$ and $S_{21}$. The material under test (MUT) was placed in a coaxial transverse electromagnetic (TEM) waves transmission line (TL). In 1974, Weir ${ }^{7}$ obtained analogous relations for the MUT placed in a rectangular waveguide. In both works the field reflection coefficient $\Gamma$ at the interface of two half-spaces and the propagation coefficient $P$ were used. They can be expressed by the above mentioned $\varepsilon$ and $\mu$.

If the sample is a solid, it is enough to consider the problem of the propagation through the slab. At least two additional layers should be considered for loose materials (e.g. synthetic granulates or grain seeds) and liquids. For this reason, the propagation in layered structure was considered.

The problem of propagation of electromagnetic (EM) waves in an anisotropic layered structure placed in the waveguide is not new or even unresolved for simple configurations. Its solution is more arduous than difficult and occurs fragmentarily in many publications. The key work seems to be article by Damaskos et al. ${ }^{8,}{ }^{9}$, describing the problem of propagation through the biaxially anisotropic slab placed in an empty rectangular waveguide. This work is surprisingly little cited in the scientific literature.

An extraction of the material parameters from scattering matrix elements $S_{11}$ and $S_{21}$ is called the inverse problem, while obtaining $S_{11}$ and $S_{21}$ from $\varepsilon$ and $\mu$ is a simple problem. The purpose of this work is to present the solution of the latter for the anisotropic material.

In our work we first derived from basics relations described transverse electric (TE) and transverse magnetic (TM) waves propagating in a rectangular waveguide filled with an anisotropic medium. Field theory methods were used ${ }^{10}$. Although this derivation is not new, it highlights the analogy and differences with propagation in an empty waveguide. Second, we discuss the transmission matrix method in anisotropic layered media highlighting the mutual relationships between the field theory and the circuit theory. It is usually not shown in elementary textbooks ${ }^{11-14}$. Third, we present scattering matrix elements $S_{11}$ and $S_{21}$ at the form known from the Nicolson-Ross-Weir model.

*andrzej.dukata@wat.edu.pl

Radioelectronic Systems Conference 2019, edited by Piotr Kaniewski, Jan Matuszewski, Proc. of SPIE Vol. 11442, 114420A · C 2020 SPIE · CCC code: 0277-786X/20/\$21 · doi: 10.1117/12.2565584 


\section{DESCRIPTION OF THE CONFIGURATION}

Consider a medium consisting of $t+2$ layers placed inside a rectangular waveguide with cross-sectional dimensions $a$ and $b$. The boundaries of the layers are perpendicular to the waveguide $z$-axis and marked as $d_{l}, l=0,1, \ldots, t$ (Fig. 1).

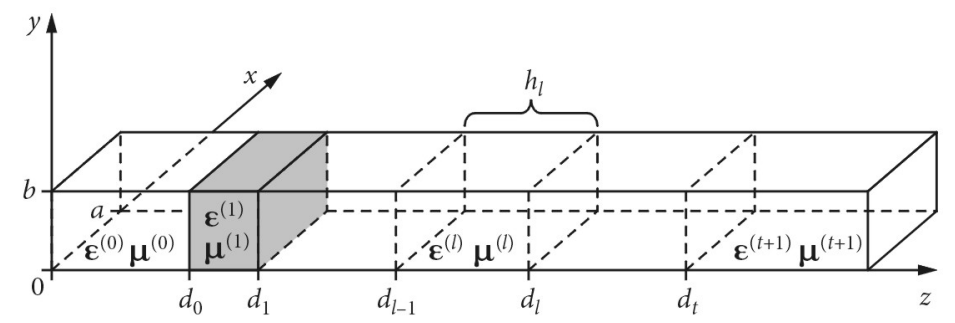

Figure 1. A layered medium placed in a rectangular waveguide with cross-sectional dimensions $a$ and $b$

Layers marked as 0 and $t+1$ have vacuum parameters. In each layer we are looking for a solution in the form of monochromatic waves propagating along the $z$-axis

$$
\begin{aligned}
\mathbf{E}^{(l)}(x, y, z, t) & =\left[\hat{\mathbf{x}} E_{0 x}^{(l)}(x, y, \omega)+\hat{\mathbf{y}} E_{0 y}^{(l)}(x, y, \omega)+\hat{\mathbf{z}} E_{0 z}^{(l)}(x, y, \omega)\right] \exp \left[\mathrm{j}\left(\omega t \mp k^{(l)} z\right)\right] \\
\mathbf{H}^{(l)}(x, y, z, t) & =\left[\hat{\mathbf{x}} H_{0 x}^{(l)}(x, y, \omega)+\hat{\mathbf{y}} H_{0 y}^{(l)}(x, y, \omega)+\hat{\mathbf{z}} H_{0 z}^{(l)}(x, y, \omega)\right] \exp \left[\mathrm{j}\left(\omega t \mp k^{(l)} z\right)\right]
\end{aligned}
$$

where $\mathbf{E}^{(l)}$ and $\mathbf{H}^{(l)}$ denotes the electric and magnetic field intensity respectively. The upper sign (minus) corresponds to the wave running in the positive and the lower sign (plus) in the negative direction of this axis. To simplify the notation, in further considerations we will skip the arguments of the function, remembering the $\exp (+\mathrm{j} \omega t)$ time dependence of all fields.

Each layer has biaxial anisotropy with principal axes parallel to the axes of the Cartesian system. In this case the magnetic flux density $\mathbf{B}^{(l)}$ and the electric flux density $\mathbf{D}^{(l)}$ are described by the constitutive relations

$$
\begin{gathered}
\mathbf{B}^{(l)}=\mu_{0} \boldsymbol{\mu}^{(l)} \mathbf{H}^{(l)} \\
\mathbf{D}^{(l)}=\varepsilon_{0} \boldsymbol{\varepsilon}^{(l)} \mathbf{E}^{(l)}
\end{gathered}
$$

and the matrices of relative permeability and magnetic permittivity tensors $\boldsymbol{\varepsilon}^{(l)}$ and $\boldsymbol{\mu}^{(l)}$ for each layer are diagonal

$$
\left[\boldsymbol{\varepsilon}^{(l)}\right]=\left[\begin{array}{ccc}
\varepsilon_{x x}^{(l)} & 0 & 0 \\
0 & \varepsilon_{y y}^{(l)} & 0 \\
0 & 0 & \varepsilon_{z z}^{(l)}
\end{array}\right] \quad\left[\boldsymbol{\mu}^{(l)}\right]=\left[\begin{array}{ccc}
\mu_{x x}^{(l)} & 0 & 0 \\
0 & \mu_{y y}^{(l)} & 0 \\
0 & 0 & \mu_{z z}^{(l)}
\end{array}\right]
$$

If two of the three parameters are the same, the medium is uniaxially anisotropic. The another parameter can be anywhere, e.g.

$$
\left[\boldsymbol{\varepsilon}^{(l)}\right]=\left[\begin{array}{ccc}
\varepsilon_{x x}^{(l)} & 0 & 0 \\
0 & \varepsilon_{x x}^{(l)} & 0 \\
0 & 0 & \varepsilon_{z z}^{(l)}
\end{array}\right] \quad\left[\boldsymbol{\mu}^{(l)}\right]=\left[\begin{array}{ccc}
\mu_{x x}^{(l)} & 0 & 0 \\
0 & \mu_{x x}^{(l)} & 0 \\
0 & 0 & \mu_{z z}^{(l)}
\end{array}\right]
$$

At this case the optic axis is along the $z$ axis. Maxwell's equations in each layer are

$$
\begin{gathered}
\nabla \times \mathbf{E}^{(l)}=-\mathrm{j} \omega \mu_{0} \boldsymbol{\mu}^{(l)} \mathbf{H}^{(l)} \\
\nabla \times \mathbf{H}^{(l)}=\mathrm{j} \omega \varepsilon_{0} \boldsymbol{\varepsilon}^{(l)} \mathbf{E}^{(l)}
\end{gathered}
$$

We assume that the waveguide walls are made of a perfect electric conductor (PEC), then the boundary conditions on the inner surface of the waveguide $S$ mean vanishing the normal component of $\mathbf{B}^{(l)}$ and the tangential components of $\mathbf{E}^{(l)}$. 
At the interface $z=d_{l}$ between the medium $l$ and $l+1$, the boundary conditions mean the continuity of the normal component of flux densities $\mathbf{B}^{(l)}$ and $\mathbf{D}^{(l)}$ and the continuity of the tangential components of field intensities $\mathbf{E}^{(l)}$ and $\mathbf{H}^{(l)}$.

Substituting the assumed form of the solution (1) and (2) into Maxwell's equations, it can be shown that the longitudinal components of the fields satisfy the coupled system of equations. Decoupling of $E_{0 z}$ from $H_{0 z}$ occurs in two particular cases (see Appendix A). In the first case $\mathrm{TE}_{m 0} \mathrm{i}_{\mathrm{TE}} \mathrm{TE}_{0 n}$ modes can exist in the layered structure with biaxial material with $\boldsymbol{\varepsilon}^{(l)}$ and $\boldsymbol{\mu}^{(l)}$ given by (5). In the second case the $\mathrm{TE}_{m n}$ modes (including $\mathrm{TE}_{m 0}$ and $\mathrm{TE}_{0 n}$ ) and $\mathrm{TM}_{m n}$ modes can propagate for specific configuration of material parameters. The latter case includes a particular form of an uniaxial anisotropy (socalled transverse isotropy) determined by (6). Wave modes result from the substitution of general solutions of decoupled wave equations into boundary conditions on the surface $S$ (see Appendix B).

Our goal is to find elements $S_{21}$ and $S_{11}$ of the scattering matrix for the EM wave propagating from the left through the layer system.

\section{SCATTERING MATRIX ELEMENTS FOR LAYERED STRUCTURE}

Let's focus on the $\mathrm{TE}_{m n}$ mode transmission through a system of layers with uniaxial anisotropy defined by (6) located in the waveguide. The field in a medium $l$ is the sum of waves running in the positive and negative direction of the $z$ axis (see Appendix 2):

$$
\begin{gathered}
H_{z}^{(l)}=A^{(l)} H_{0 z}^{(l)} \exp \left[\mathrm{j}\left(\omega t-k^{(l)} z\right)\right]+B^{(l)} H_{0 z}^{(l)} \exp \left[\mathrm{j}\left(\omega t+k^{(l)} z\right)\right] \\
E_{x}^{(l)}=A^{(l)} E_{0 x}^{(l)} \exp \left[\mathrm{j}\left(\omega t-k^{(l)} z\right)\right]+B^{(l)} E_{0 x}^{(l)} \exp \left[\mathrm{j}\left(\omega t+k^{(l)} z\right)\right] \\
H_{y}^{(l)}=\frac{1}{Z_{\mathrm{TE}}^{(l)}}\left[A^{(l)} E_{0 x}^{(l)} \exp \left[\mathrm{j}\left(\omega t-k^{(l)} z\right)\right]-B^{(l)} E_{0 x}^{(l)} \exp \left[\mathrm{j}\left(\omega t+k^{(l)} z\right)\right]\right] \\
E_{y}^{(l)}=A^{(l)} E_{0 y}^{(l)} \exp \left[\mathrm{j}\left(\omega t-k^{(l)} z\right)\right]+B^{(l)} E_{0 y}^{(l)} \exp \left[\mathrm{j}\left(\omega t+k^{(l)} z\right)\right] \\
H_{x}^{(l)}=-\frac{1}{Z_{\mathrm{TE}}^{(l)}}\left[A^{(l)} E_{0 y}^{(l)} \exp \left[\mathrm{j}\left(\omega t-k^{(l)} z\right)\right]-B^{(l)} E_{0 y}^{(l)} \exp \left[\mathrm{j}\left(\omega t+k^{(l)} z\right)\right]\right]
\end{gathered}
$$

where

$$
\begin{gathered}
H_{0 z}^{(l)}=\cos \left(\frac{m \pi}{a} x\right) \cos \left(\frac{n \pi}{b} y\right) \\
E_{0 x}^{(l)}=\frac{\mathrm{j} \omega \mu_{0} \mu_{z z}^{(l)}}{k_{m n}^{2}} \frac{n \pi}{b} \cos \left(\frac{m \pi}{a} x\right) \sin \left(\frac{n \pi}{b} y\right) \\
E_{0 y}^{(l)}=-\frac{\mathrm{j} \omega \mu_{0} \mu_{z z}^{(l)}}{k_{m n}^{2}} \frac{m \pi}{a} \sin \left(\frac{m \pi}{a} x\right) \cos \left(\frac{n \pi}{b} y\right) \\
k^{(l)}=k_{0} \sqrt{\mu_{x x}^{(l)} \varepsilon_{x x}^{(l)}} \sqrt{1-\frac{1}{\varepsilon_{x x}^{(l)}} \mu_{z z}^{(l)}} \frac{k_{m n}^{2}}{k_{0}^{2}} \\
Z_{\mathrm{TE}}^{(l)}=\frac{\omega \mu_{0} \mu_{x x}^{(l)}}{k^{(l)}} \\
k_{0}=\omega \sqrt{\mu_{0} \varepsilon_{0}}=\frac{\omega}{c} \\
k_{m n}=\pi \sqrt{\left(\frac{m}{a}\right)^{2}+\left(\frac{n}{b}\right)^{2}}
\end{gathered}
$$


Boundary conditions at the interface $z=d_{l}$ require continuity of the normal component of flux densities and the tangential components of field intensities. Relations between the amplitudes $A^{(l)}$ and $B^{(l)}$ can be obtained by using boundary conditions continuity of fields $E_{x}$ and $H_{y}$ only:

$$
E_{x}^{(l)}\left(z=d_{1}\right)=E_{x}^{(l+1)}\left(z=d_{1}\right), \quad H_{y}^{(l)}\left(z=d_{l}\right)=H_{y}^{(l+1)}\left(z=d_{l}\right)
$$

It may be shown that other three equations are not independent. Substituting (9) and (10) to (11) we get

$$
\begin{gathered}
\left.\left.\frac{\mu_{z z}^{(l)}}{\mu_{z z}^{(l+1)}} A^{(l)} \exp \left(-\mathrm{j} k^{(1)} d_{l}\right)+B^{(l)} \exp \left[\mathrm{j} k^{(1)} d_{l}\right)\right]=A^{(l+1)} \exp \left(-\mathrm{j} k^{(l+1)} d_{l}\right)+B^{(l+1)} \exp \left[\mathrm{j} k^{(l+1)} d_{l}\right)\right] \\
\frac{Z_{\mathrm{TE}}^{(l+1)}}{Z_{\mathrm{TE}}^{(l)}} \frac{\mu_{z z}^{(l)}}{\mu_{z z}^{(l+1)}}\left[A^{(l)} \exp \left(-\mathrm{j} k^{(l)} d_{l}\right)-B^{(l)} \exp \left(\mathrm{j} k^{(l)} d_{l}\right)\right]=\left[A^{(l+1)} \exp \left(-\mathrm{j} k^{(l+1)} d_{l}\right)-B^{(l+1)} \exp \left(\mathrm{j} k^{(l+1)} d_{l}\right)\right]
\end{gathered}
$$

The above system of equations can be written in the matrix form (see Appendix C)

$$
\left[\begin{array}{l}
A^{(l+1)} \\
B^{(l+1)}
\end{array}\right]=\left[\begin{array}{cc}
\mathrm{e}^{\mathrm{j} k^{(l+1)} d_{l}} & 0 \\
0 & \mathrm{e}^{-\mathrm{j} k^{(l+1)} d_{l}}
\end{array}\right] \mathbf{W}^{(l, l+1)}\left[\begin{array}{cc}
\mathrm{e}^{-\mathrm{j} k^{(l)} d_{l}} & 0 \\
0 & \mathrm{e}^{\mathrm{j} k^{(l)} d_{l}}
\end{array}\right]\left[\begin{array}{l}
A^{(l)} \\
B^{(l)}
\end{array}\right]
$$

where

$$
\mathbf{W}^{(l, l+1)}=\frac{\mu_{z z}^{(l)}}{\mu_{z z}^{(l+1)}} \frac{1}{2}\left[\begin{array}{ll}
1+Z^{(l, l+1)} & 1-Z^{(l, l+1)} \\
1-Z^{(l, l+1)} & 1+Z^{(l, l+1)}
\end{array}\right]
$$

and

$$
Z^{(l, l+1)}=\frac{Z_{\mathrm{TE}}^{(l+1)}}{Z_{\mathrm{TE}}^{(l)}}
$$

An Eq. (15), described the matrix $\mathbf{W}^{(l, l+I)}$, is a generalization of the Eq. (12) presented in the Rulf $f^{15}$ paper. That work concerned with the isotropic medium.

Let's write the following expressions based on (14)

$$
\begin{aligned}
& {\left[\begin{array}{l}
A^{(1)} \\
B^{(1)}
\end{array}\right]=\left[\begin{array}{cc}
\mathrm{e}^{\mathrm{j} k^{(1)} d_{0}} & 0 \\
0 & \mathrm{e}^{-\mathrm{jk} k^{(1)} d_{0}}
\end{array}\right] \mathbf{W}^{(0,1)}\left[\begin{array}{cc}
\mathrm{e}^{-\mathrm{j} k^{(0)} d_{0}} & 0 \\
0 & \mathrm{e}^{\mathrm{j} k^{(0)} d_{0}}
\end{array}\right]\left[\begin{array}{l}
A^{(0)} \\
B^{(0)}
\end{array}\right]} \\
& {\left[\begin{array}{l}
A^{(2)} \\
B^{(2)}
\end{array}\right]=\left[\begin{array}{cc}
\mathrm{e}^{\mathrm{j} k^{(2)} d_{1}} & 0 \\
0 & \mathrm{e}^{-\mathrm{j} k^{(2)} d_{1}}
\end{array}\right] \mathbf{W}^{(1,2)}\left[\begin{array}{cc}
\mathrm{e}^{-\mathrm{j} k^{(1)} d_{1}} & 0 \\
0 & \mathrm{e}^{\mathrm{j} k^{(1)} d_{1}}
\end{array}\right]\left[\begin{array}{l}
A^{(1)} \\
B^{(1)}
\end{array}\right]} \\
& {\left[\begin{array}{l}
A^{(3)} \\
B^{(3)}
\end{array}\right]=\left[\begin{array}{cc}
\mathrm{e}^{\mathrm{j} k^{(3)} d_{2}} & 0 \\
0 & \mathrm{e}^{-\mathrm{j} k^{(3)} d_{2}}
\end{array}\right] \mathbf{W}^{(2,3)}\left[\begin{array}{cc}
\mathrm{e}^{-\mathrm{j} k^{(2)} d_{2}} & 0 \\
0 & \mathrm{e}^{\mathrm{j} k^{(2)} d_{2}}
\end{array}\right]\left[\begin{array}{l}
A^{(2)} \\
B^{(2)}
\end{array}\right]}
\end{aligned}
$$

Substituting (16) to (17) we obtain

$$
\left[\begin{array}{l}
A^{(2)} \\
B^{(2)}
\end{array}\right]=\left[\begin{array}{cc}
\mathrm{e}^{\mathrm{j} k^{(2)} d_{1}} & 0 \\
0 & \mathrm{e}^{-\mathrm{j} k^{(2)} d_{1}}
\end{array}\right] \mathbf{W}^{(1,2)}\left[\begin{array}{cc}
\mathrm{e}^{-\mathrm{j} k^{(1)}\left(d_{1}-d_{0}\right)} & 0 \\
0 & \mathrm{e}^{\mathrm{j} k^{(1)}\left(d_{1}-d_{0}\right)}
\end{array}\right] \mathbf{W}^{(0,1)}\left[\begin{array}{cc}
\mathrm{e}^{-\mathrm{j} k^{(0)} d_{0}} & 0 \\
0 & \mathrm{e}^{\mathrm{j} k^{(0)} d_{0}}
\end{array}\right]\left[\begin{array}{l}
A^{(0)} \\
B^{(0)}
\end{array}\right]
$$

and finally

$$
\left[\begin{array}{l}
A^{(3)} \\
B^{(3)}
\end{array}\right]=\left[\begin{array}{cc}
\mathrm{e}^{\mathrm{j} k^{(3)} d_{2}} & 0 \\
0 & \mathrm{e}^{-\mathrm{j} k^{(3)} d_{2}}
\end{array}\right] \mathbf{W}^{(2,3)}\left[\begin{array}{cc}
\mathrm{e}^{-\mathrm{j} k^{(2)}\left(d_{2}-d_{1}\right)} & 0 \\
0 & \mathrm{e}^{\mathrm{j} k^{(2)}\left(d_{2}-d_{1}\right)}
\end{array}\right] \mathbf{W}^{(1,2)}\left[\begin{array}{cc}
\mathrm{e}^{-\mathrm{j} k^{(1)}\left(d_{1}-d_{0}\right)} & 0 \\
0 & \mathrm{e}^{\mathrm{j} k^{(1)}\left(d_{1}-d_{0}\right)}
\end{array}\right] \mathbf{W}^{(0,1)}\left[\begin{array}{cc}
\mathrm{e}^{-\mathrm{j} k^{(0)} d_{0}} & 0 \\
0 & \mathrm{e}^{\mathrm{j} k^{(0)} d_{0}}
\end{array}\right]\left[\begin{array}{l}
A^{(0)} \\
B^{(0)}
\end{array}\right]
$$


Let's denote $h_{0}=d_{0}$ and the widths of particular layers as $h_{l}$

$$
h_{l}=d_{l}-d_{l-1}, l=1, \ldots, t
$$

One can then define

$$
\mathbf{P}^{(l)}=\left[\begin{array}{cc}
\mathrm{e}^{-\mathrm{j} k^{(l)} h_{l}} & 0 \\
0 & \mathrm{e}^{\mathrm{j} k^{(l)} h_{l}}
\end{array}\right]
$$

For a structure composed of $t+2$ layers (see Fig. 1) we obtain

$$
\left[\begin{array}{l}
A^{(t+1)} \\
B^{(t+1)}
\end{array}\right]=\left[\begin{array}{cc}
\mathrm{e}^{\mathrm{j} k^{(t+1)} d_{t}} & 0 \\
0 & \mathrm{e}^{-\mathrm{j} k^{(t+1)} d_{t}}
\end{array}\right] \mathbf{W}\left[\begin{array}{l}
A^{(0)} \\
B^{(0)}
\end{array}\right]
$$

where

$$
\mathbf{W}=\prod_{l=0}^{t} \mathbf{W}^{(l, l+1)} \mathbf{P}^{(l)}
$$

To obtain the $S_{21}$ and $S_{11}$ scattering matrix elements, one must find the relations of the amplitudes $A^{(t+1)}$ and $B^{(t+1)}$ with the notation $A_{t+1}$ and $B_{t+1}$ used in the theory of TL for the same reference plane $z=d_{t}$. The amplitudes $A_{0}$ and $B_{0}$ at point $z=0$ on a uniform TL are related to the amplitudes $A_{t+1}$ and $B_{t+1}$ at point $z=d_{t}$ on the same line (see e.g. Rulf ${ }^{15}$, Eq. (13))

$$
\left[\begin{array}{l}
A_{0} \\
B_{0}
\end{array}\right]=\left[\begin{array}{cc}
\mathrm{e}^{\mathrm{j} k^{(0)} d_{t}} & 0 \\
0 & \mathrm{e}^{-\mathrm{j} k^{(0)} d_{t}}
\end{array}\right]\left[\begin{array}{l}
A_{t+1} \\
B_{t+1}
\end{array}\right]
$$

If $d_{0}=0$, then $A^{(0)}=A_{0}$ and $B^{(0)}=B_{0}$. Then $d_{t}$ is the sum of the widths of all the layers. Hence

$$
\left[\begin{array}{l}
A^{(t+1)} \\
B^{(t+1)}
\end{array}\right]=\left[\begin{array}{cc}
\mathrm{e}^{\mathrm{j} k^{(0)} d_{t}} & 0 \\
0 & \mathrm{e}^{-\mathrm{j} k^{(0)} d_{t}}
\end{array}\right]\left[\begin{array}{l}
A_{t+1} \\
B_{t+1}
\end{array}\right]
$$

The expression (20) can then be written as

$$
\left[\begin{array}{c}
A_{t+1} \\
B_{t+1}
\end{array}\right]=\mathbf{W}\left[\begin{array}{l}
A_{0} \\
B_{0}
\end{array}\right]
$$

The scattering matrix elements $S_{21}$ and $S_{11}$ satisfy following relationships

$$
B_{0}=S_{11} A_{0}, \quad A_{t+1}=S_{21} A_{0}
$$

If the wave is incident from the left, then $B_{t+1}=0$. We obtain

$$
\left[\begin{array}{c}
S_{21} \\
0
\end{array}\right]=\mathbf{W}\left[\begin{array}{c}
1 \\
S_{11}
\end{array}\right]
$$

Next, if we denote elements of the $\mathbf{W}$ matrix as

$$
\mathbf{W}=\left(\begin{array}{ll}
w_{11} & w_{12} \\
w_{21} & w_{22}
\end{array}\right)
$$

we get the scattering matrix elements $S_{21}$ and $S_{11}$ of the layer system:

$$
S_{21}=\frac{w_{11} w_{22}-w_{12} w_{21}}{w_{22}}=\frac{|\mathbf{W}|}{w_{22}}, \quad S_{11}=-\frac{w_{21}}{w_{22}}
$$




\section{AN EXAMPLE: SCATTERING MATRIX ELEMENTS OF A SINGLE SLAB}

As an example, we examine the transmission through a single slab placed in an empty waveguide. We shall find a relation between the amplitudes on the two sides of an interface, first. We may assume without loss of generality that there is an interface $z=d_{0}=0$ between layer 0 (to the left) and 1 (to the right). Then

$$
\begin{gathered}
{\left[\begin{array}{l}
A^{(1)} \\
B^{(1)}
\end{array}\right]=\mathbf{W}^{(0,1)}\left[\begin{array}{l}
A^{(0)} \\
B^{(0)}
\end{array}\right]} \\
\mathbf{W}^{(0,1)}=\frac{\mu_{z z}^{(0)}}{\mu_{z z}^{(1)}} \frac{1}{2}\left[\begin{array}{ll}
1+Z^{(0,1)} & 1-Z^{(0,1)} \\
1-Z^{(0,1)} & 1+Z^{(0,1)}
\end{array}\right]
\end{gathered}
$$

Assuming that in the area 1 there is no wave returning from infinity, then $B^{(1)}=0$. We define the reflection coefficient $\Gamma$ and the transmission coefficient $T$ at the interface as (see e.g. Balanis ${ }^{12}$, p. 181):

$$
B^{(0)}=\Gamma A^{(0)}, A^{(1)}=T A^{(0)}
$$

After some manipulations we obtain

$$
\begin{gathered}
\Gamma=\frac{Z^{(0,1)}-1}{Z^{(0,1)}+1} \\
T=(1+\Gamma) \frac{\mu_{z z}^{(0)}}{\mu_{z z}^{(1)}}
\end{gathered}
$$

We will consider transmission through layer 1 assuming that media denoted by 0 and 2 have vacuum parameters. In addition, for simplicity we denote $d_{1}=d$. From (19), formally

$$
\left[\begin{array}{l}
A^{(2)} \\
B^{(2)}
\end{array}\right]=\left[\begin{array}{cc}
\mathrm{e}^{\mathrm{j} k^{(2)} d} & 0 \\
0 & \mathrm{e}^{-\mathrm{j} k^{(2)} d}
\end{array}\right] \mathbf{W}^{(1,2)}\left[\begin{array}{cc}
\mathrm{e}^{-\mathrm{j} k^{(1)} d} & 0 \\
0 & \mathrm{e}^{\mathrm{j} k^{(1)} d}
\end{array}\right] \mathbf{W}^{(0,1)}\left[\begin{array}{l}
A^{(0)} \\
B^{(0)}
\end{array}\right]
$$

Taking into account that $k^{(2)}=k^{(0)}$ and $\mathbf{W}^{(1,2)}=\mathbf{W}^{(1,0)}$ then the above formula takes the form

$$
\left[\begin{array}{l}
A^{(2)} \\
B^{(2)}
\end{array}\right]=\left[\begin{array}{cc}
\mathrm{e}^{\mathrm{j} k^{(0)} d} & 0 \\
0 & \mathrm{e}^{-\mathrm{j} k^{(0)} d}
\end{array}\right] \mathbf{W}\left[\begin{array}{l}
A^{(0)} \\
B^{(0)}
\end{array}\right]
$$

or using (22)

$$
\left[\begin{array}{l}
A_{2} \\
B_{2}
\end{array}\right]=\mathbf{W}\left[\begin{array}{l}
A_{0} \\
B_{0}
\end{array}\right]
$$

The matrix $\mathbf{W}$ can be rewritten as

$$
\mathbf{W}=\mathbf{W}^{(1,0)}\left[\begin{array}{cc}
P & 0 \\
0 & P^{-1}
\end{array}\right] \mathbf{W}^{(0,1)}
$$

where the letter $P$ indicates propagation factor $^{7}$

$$
P=\exp \left(-\mathrm{j} k^{(1)} d\right)
$$

After some manipulations matrices $\mathbf{W}^{(1,0)}$ and $\mathbf{W}^{(0,1)}$ can be expressed by the reflection coefficient at the interface $\Gamma$

$$
\mathbf{W}^{(1,0)}=\frac{\mu_{z z}^{(1)}}{\mu_{z z}^{(0)}} \frac{1}{1+\Gamma}\left[\begin{array}{cc}
1 & \Gamma \\
\Gamma & 1
\end{array}\right], \quad \mathbf{W}^{(0,1)}=\frac{\mu_{z}^{(0)}}{\mu_{z z}^{(1)}} \frac{1}{1-\Gamma}\left[\begin{array}{cc}
1 & -\Gamma \\
-\Gamma & 1
\end{array}\right]
$$


Finally $\mathbf{W}$ takes the form

$$
\mathbf{W}=\frac{1}{\left(1-\Gamma^{2}\right) P}\left[\begin{array}{cc}
P^{2}-\Gamma^{2} & \Gamma\left(1-P^{2}\right) \\
-\Gamma\left(1-P^{2}\right) & 1-\Gamma^{2} P^{2}
\end{array}\right]
$$

It can be shown that $\mathbf{W}$ is an unimodular matrix (see e.g. Born and Wolf $f^{13}$, p. 60). This means that the determinant of the matrix is equal to unity.

Repeating the arguments cited above $B_{2}=0, B_{0}=S_{11} A_{0}$ and $A_{2}=S_{21} A_{0}$. From (23)

$$
S_{21}=\frac{|\mathbf{W}|}{w_{22}}=\frac{1}{w_{22}}, \quad S_{11}=-\frac{w_{21}}{w_{22}}
$$

or

$$
S_{21}=\frac{P\left(1-\Gamma^{2}\right)}{1-\Gamma^{2} P^{2}}, \quad S_{11}=\frac{\Gamma\left(1-P^{2}\right)}{1-\Gamma^{2} P^{2}}
$$

The form (28) of obtained scattering matrix elements $S_{21}$ and $S_{11}$ of the layer with uniaxial anisotropy is consistent with the initial formulas of the NRW method ${ }^{6,7}$ of the isotropic layer, derived using the method of graphs.

\section{REMARKS ON OTHER CONSIDERED WAVE MODES}

It is not difficult to notice that equations (28) are also valid for other considered cases. In the expression for $P$, should be substituted the appropriate wave number $k^{(1)}$ and in the formula for $\Gamma$ - the corresponding impedance quotient $Z^{(0,1)}$. These parameters are summarized in the Table 1 and the Table 2. Material parameters affecting on EM wave propagation

\begin{tabular}{|c|c|}
\hline $\begin{array}{c}\mathrm{TE}_{m n} \text { modes; } \\
\text { parameter set }\left(\varepsilon_{x x}, \mu_{x x}, \mu_{z z}\right)\end{array}$ & $\begin{array}{c}\mathrm{TM}_{m n} \text { modes; } \\
\text { parameter set }\left(\varepsilon_{x x}, \mu_{x x}, \varepsilon_{z z}\right)\end{array}$ \\
\hline$\Gamma=\frac{Z^{(0,1)}-1}{Z^{(0,1)}+1}$ & \\
\hline$Z^{(0,1)}=\frac{Z_{\mathrm{TE}}^{(1)}}{Z_{\mathrm{TE}}^{(0)}}$ & $Z^{(0,1)}=\frac{Z_{\mathrm{TM}}^{(1)}}{Z_{\mathrm{TM}}^{(0)}}$ \\
\hline$Z_{\mathrm{TE}}^{(l)}=\eta \frac{\sqrt{\frac{\mu_{x x}^{(l)}}{\varepsilon_{x x}^{(l)}}}}{\sqrt{1-\frac{1}{\varepsilon_{x x}^{(l)} \mu_{z z}^{(l)}} \frac{k_{m n}^{2}}{k_{0}^{2}}}}$ & $Z_{\mathrm{TM}}^{(l)}=\eta \sqrt{\frac{\mu_{x x}^{(l)}}{\varepsilon_{x x}^{(l)}}} \sqrt{1-\frac{1}{\mu_{x x}^{(l)} \varepsilon_{z z}^{(l)}} \frac{k_{m n}^{2}}{k_{0}^{2}}}$ \\
\hline \multicolumn{2}{|l|}{$P=\exp \left(-\mathrm{j} k^{(1)} d\right)$} \\
\hline$k^{(1)}=k_{0} \sqrt{\mu_{x x}^{(1)} \varepsilon_{x x}^{(1)}} \sqrt{1-\frac{1}{\varepsilon_{x x}^{(1)} \mu_{z z}^{(1)}} \frac{k_{m n}^{2}}{k_{0}^{2}}}$ & $k^{(1)}=k_{0} \sqrt{\mu_{x x}^{(1)} \varepsilon_{x x}^{(1)}} \sqrt{1-\frac{1}{\mu_{x x}^{(1)} \varepsilon_{z z}^{(1)}} \frac{k_{m n}^{2}}{k_{0}^{2}}}$ \\
\hline$k_{m n}=\pi \sqrt{\left(\frac{m}{a}\right)^{2}+\left(\frac{n}{b}\right)^{2}}$ & \\
\hline
\end{tabular}
are given in brackets in the table head.

Table 1. Parameters $\Gamma$ and $P$ of the $\mathrm{TE}_{m n}$ and $\mathrm{TM}_{m n}$ modes propagation through an uniaxially anisotropic slab 
Table 2. Parameters $\Gamma$ and $P$ of the $\mathrm{TE}_{m 0}$ and $\mathrm{TE}_{0 n}$ modes propagation through a biaxially anisotropic slab

\begin{tabular}{|c|c|}
\hline $\begin{array}{c}\mathrm{TE}_{m 0} \text { modes } \\
\text { parameter set }\left(\varepsilon_{y y}, \mu_{x x}, \mu_{z z}\right)\end{array}$ & $\begin{array}{c}\mathrm{TE}_{0 n} \text { modes } \\
\text { parameter set }\left(\varepsilon_{x x}, \mu_{y y}, \mu_{z z}\right)\end{array}$ \\
\hline \multicolumn{2}{|l|}{$\Gamma=\frac{Z^{(0,1)}-1}{Z^{(0,1)}+1}$} \\
\hline$Z^{(0,1)}=\frac{Z_{m 0}^{(1)}}{Z_{m 0}^{(0)}}$ & $Z^{(0,1)}=\frac{Z_{0 n}^{(1)}}{Z_{0 n}^{(0)}}$ \\
\hline$Z_{m 0}^{(l)}=\eta \frac{\sqrt{\frac{\mu_{x x}^{(l)}}{\varepsilon_{y y}^{(l)}}}}{\sqrt{1-\frac{1}{\varepsilon_{y y}^{(l)} \mu_{z z}^{(l)}} \frac{k_{m 0}^{2}}{k_{0}^{2}}}}$ & $Z_{0 n}^{(l)}=\eta \frac{\sqrt{\frac{\mu_{y y}^{(l)}}{\varepsilon_{x x}^{(l)}}}}{\sqrt{1-\frac{1}{\varepsilon_{x x}^{(l)} \mu_{z z}^{(l)}} \frac{k_{0 n}^{2}}{k_{0}^{2}}}}$ \\
\hline \multicolumn{2}{|l|}{$P=\exp \left(-\mathrm{j} k^{(1)} d\right)$} \\
\hline$k^{(1)}=k_{0} \sqrt{\mu_{x x}^{(1)} \varepsilon_{y y}^{(1)}} \sqrt{1-\frac{1}{\varepsilon_{y y}^{(1)} \mu_{z z}^{(1)}} \frac{k_{m 0}^{2}}{k_{0}^{2}}}$ & $k^{(1)}=k_{0} \sqrt{\mu_{y y}^{(1)} \varepsilon_{x x}^{(1)}} \sqrt{1-\frac{1}{\varepsilon_{x x}^{(1)} \mu_{z z}^{(1)}} \frac{k_{m n}^{2}}{k_{0}^{2}}}$ \\
\hline$k_{m 0}=\frac{m \pi}{a}$ & $k_{0 n}=\frac{n \pi}{b}$ \\
\hline
\end{tabular}

\section{CONCLUSION}

We have derived a formalism and presented a solution of the boundary value problem of EM waves transmission through an anisotropic layered structure placed in a rectangular waveguide. The main axes of the crystallographic system and the Cartesian system were oriented parallel to the waveguide edges. Hybrid modes as well as TE and TM may exist in this configuration, with TM waves only for an uniaxial anisotropy of a special form (so-called transverse isotropy). For the most general biaxial anisotropy, only $\mathrm{TE}_{m 0}$ and $\mathrm{TE}_{0 n}$ waves can exist ${ }^{8}$.

The $\mathrm{TE}_{m n}$ mode transmission through a system of uniaxially anisotropic layers was considered in detail. We found the elements $S_{21}$ and $S_{11}$ of the scattering matrix for the EM wave propagating from the left. The resulting matrix $\mathbf{W}$ (see Eq. (21)), derived in this paper, plays a crucial role in determining $S_{21}$ and $S_{11}$. As a matter of fact, the form of matrix $\mathbf{W}$ is the same as reported by other authors for the isotropic ${ }^{11}$ and electrically anisotropic ${ }^{5}$ media. The novelty is obtaining new relations for wave impedances and wave numbers assigned to the individual layers. For other cases, considered in the Appendices A and B, these relationships are shown in Table 1 and 2. Appropriate parameters for isotropic media, known from the circuits theory ${ }^{11}$, should be replaced by these expressions.

In the important case of transmission through a single slab, the solutions in the form of the $S_{11}$ and $S_{21}$ elements of the scattering matrix are obtained and presented in the same form as in the NRW method ${ }^{6-7}$. This enables their direct application for the extraction of complex components of permittivity and permeability tensors.

In the general case it is possible to determine as many material parameters as many independent measurement results (in general, complex) we have. For the isotropy, the complex parameters $S_{21}$ and $S_{11}$ correspond to the complex permittivity $\varepsilon$ and permeability $\mu$. It is possible to extract both materials parameters using the scattering matrix elements by the only measurement in a specific configuration. In the case of anisotropy, it is clear that an additional one (see Table 1) or two (see Table 2) measurements should be made in a different configuration. For example, the MUT should be prepared differently (e.g. rotated ${ }^{5}$, placed in another waveguide ${ }^{16}$ ) or a different mode should be used ${ }^{8}$.

In addition to the LHM and CFRP structures mentioned earlier, the results can be utilized to study classic anisotropic materials with known orientation of the main axes and man-made material structures, in particular artificial dielectrics ${ }^{17}$, magnetodielectrics ${ }^{18}$ and other layered composites, characterized by transverse isotropy of effective parameters. 


\section{APPENDIX A. TE AND TM WAVES IN AN ANISOTROPIC MATERIAL}

In each layer substituting (1) and (2) into Maxwell's equations (7) and (8), we obtain

$$
\begin{gathered}
\frac{\partial E_{0 z}}{\partial y} \pm \mathrm{j} k E_{0 y}=-\mathrm{j} \omega \mu_{0} \mu_{x x} H_{0 x} \\
\mp \mathrm{j} k E_{0 x}-\frac{\partial E_{0 z}}{\partial x}=-\mathrm{j} \omega \mu_{0} \mu_{y y} H_{0 y} \\
\frac{\partial E_{0 y}}{\partial x}-\frac{\partial E_{0 x}}{\partial y}=-\mathrm{j} \omega \mu_{0} \mu_{z z} H_{0 z} \\
\frac{\partial H_{0 z}}{\partial y} \pm \mathrm{j} k H_{0 y}=\mathrm{j} \omega \varepsilon_{0} \varepsilon_{x x} E_{0 x} \\
\mp \mathrm{j} k H_{0 x}-\frac{\partial H_{0 z}}{\partial x}=\mathrm{j} \omega \varepsilon_{0} \varepsilon_{y y} E_{0 y} \\
\frac{\partial H_{0 y}}{\partial x}-\frac{\partial H_{0 x}}{\partial y}=\mathrm{j} \omega \varepsilon_{0} \varepsilon_{z z} E_{0 z}
\end{gathered}
$$

Let's we write (29) and (33) in the form of a system of equations

$$
\left\{\begin{array}{l} 
\pm \mathrm{j} k E_{0 y}+\mathrm{j} \omega \mu_{0} \mu_{x x} H_{0 x}=-\frac{\partial E_{0 z}}{\partial y} \\
-\mathrm{j} \omega \varepsilon_{0} \varepsilon_{y y} E_{0 y} \mp \mathrm{j} k H_{0 x}=\frac{\partial H_{0 z}}{\partial x}
\end{array}\right.
$$

and determine $E_{0 y}$ and $H_{0 x}$ with respect to partial derivatives of longitudinal components $E_{0 z}$ and $H_{0 z}$. We get

$$
\begin{aligned}
& E_{0 y}=\frac{\mathrm{j}}{k_{0}^{2} \mu_{x x} \varepsilon_{y y}-k^{2}}\left(\omega \mu_{0} \mu_{x x} \frac{\partial H_{0 z}}{\partial x} \mp k \frac{\partial E_{0 z}}{\partial y}\right) \\
& H_{0 x}=\frac{\mathrm{j}}{k_{0}^{2} \mu_{x x} \varepsilon_{y y}-k^{2}}\left(\omega \varepsilon_{0} \varepsilon_{y y} \frac{\partial E_{0 z}}{\partial y} \mp k \frac{\partial H_{0 z}}{\partial x}\right)
\end{aligned}
$$

where $k_{0}$ is the wave number in the vacuum

$$
k_{0}=\omega \sqrt{\mu_{0} \varepsilon_{0}}=\frac{\omega}{c}
$$

In the same way from (30) and (32) can be obtained $E_{0 x}$ and $H_{0 y}$ relative to the partial derivatives of $E_{0 z}$ and $H_{0 z}$

$$
\begin{aligned}
& H_{0 y}=\frac{\mathrm{j}}{k_{0}^{2} \varepsilon_{x x} \mu_{y y}-k^{2}}\left(-\omega \varepsilon_{0} \varepsilon_{x x} \frac{\partial E_{0 z}}{\partial x} \mp k \frac{\partial H_{0 z}}{\partial y}\right) \\
& E_{0 x}=\frac{\mathrm{j}}{k_{0}^{2} \varepsilon_{x x} \mu_{y y}-k^{2}}\left(-\omega \mu_{0} \mu_{y y} \frac{\partial H_{0 z}}{\partial y} \mp k \frac{\partial E_{0 z}}{\partial x}\right)
\end{aligned}
$$

Substituting $E_{0 x}$ and $E_{0 y}$ into (40) and $H_{0 x}$ and $H_{0 y}$ into (43) we get the system of coupled equations for $E_{0 z}$ and $H_{0 z}$

$$
\frac{1}{k_{0}^{2} \mu_{x x} \varepsilon_{y y}-k^{2}}\left(\omega \mu_{0} \mu_{x x} \frac{\partial^{2} H_{0 z}}{\partial x^{2}} \mp k \frac{\partial^{2} E_{0 z}}{\partial x \partial y}\right)-\frac{1}{k_{0}^{2} \varepsilon_{x x} \mu_{y y}-k^{2}}\left(-\omega \mu_{0} \mu_{y y} \frac{\partial^{2} H_{0 z}}{\partial y^{2}} \mp k \frac{\partial^{2} E_{0 z}}{\partial y \partial x}\right)=-\omega \mu_{0} \mu_{z z} H_{0 z}
$$




$$
\frac{1}{k_{0}^{2} \varepsilon_{x x} \mu_{y y}-k^{2}}\left(-\omega \varepsilon_{0} \varepsilon_{x x} \frac{\partial^{2} E_{0 z}}{\partial x^{2}} \mp k \frac{\partial^{2} H_{0 z}}{\partial x \partial y}\right)-\frac{1}{k_{0}^{2} \mu_{x x} \varepsilon_{y y}-k^{2}}\left(\omega \varepsilon_{0} \varepsilon_{y y} \frac{\partial^{2} E_{0 z}}{\partial y^{2}} \mp k \frac{\partial^{2} H_{0 z}}{\partial y \partial x}\right)=\omega \varepsilon_{0} \varepsilon_{z z} E_{0 z}
$$

Each of equations (41) and (42) is a second order partial differential equation with constant coefficients of two functions $E_{0 z}(x, y)$ and $H_{0 z}(x, y)$. In general, hybrid waves propagation should be expected, which from the point of view of extracting parameters is not convenient. Instead, let's explore the possibility of TE and TM waves propagation.

We substitute $E_{0 z}=0$ to (41) and (42), hence for TE waves

$$
\begin{gathered}
\frac{\mu_{x x}}{k_{0}^{2} \mu_{x x} \varepsilon_{y y}-k^{2}}\left(\frac{\partial^{2} H_{0 z}}{\partial x^{2}}\right)+\frac{\mu_{y y}}{k_{0}^{2} \varepsilon_{x x} \mu_{y y}-k^{2}}\left(\frac{\partial^{2} H_{0 z}}{\partial y^{2}}\right)+\mu_{z z} H_{0 z}=0 \\
\frac{k_{0}^{2}\left(\mu_{x x} \varepsilon_{y y}-\varepsilon_{x x} \mu_{y y}\right)}{\left(k_{0}^{2} \varepsilon_{x x} \mu_{y y}-k^{2}\right)\left(k_{0}^{2} \mu_{x x} \varepsilon_{y y}-k^{2}\right)}\left(\frac{\partial^{2} H_{0 z}}{\partial x \partial y}\right)=0
\end{gathered}
$$

Similarly, for TM waves we have $H_{0 z}=0$, i.e.

$$
\begin{gathered}
\frac{k_{0}^{2}\left(\varepsilon_{x x} \mu_{y y}-\mu_{x x} \varepsilon_{y y}\right)}{\left(k_{0}^{2} \mu_{x x} \varepsilon_{y y}-k^{2}\right)\left(k_{0}^{2} \varepsilon_{x x} \mu_{y y}-k^{2}\right)}\left(\frac{\partial^{2} E_{0 z}}{\partial x \partial y}\right)=0 \\
\frac{\varepsilon_{x x}}{k_{0}^{2} \varepsilon_{x x} \mu_{y y}-k^{2}}\left(\frac{\partial^{2} E_{0 z}}{\partial x^{2}}\right)+\frac{\varepsilon_{y y}}{k_{0}^{2} \mu_{x x} \varepsilon_{y y}-k^{2}}\left(\frac{\partial^{2} E_{0 z}}{\partial y^{2}}\right)+\varepsilon_{z z} E_{0 z}=0
\end{gathered}
$$

Decoupling of $E_{0 z}$ from $H_{0 z}$, i.e. obtaining separate equations for $E_{0 z}$ and $H_{0 z}$, occurs in two particular cases. In the first case the following relation occurs: $\mu_{x x} \varepsilon_{y y}-\varepsilon_{x x} \mu_{y y}=0$. Rewrite it as

$$
\frac{\varepsilon_{y y}}{\varepsilon_{x x}}=\frac{\mu_{y y}}{\mu_{x x}}=\alpha
$$

This condition resets equation (44) for TE waves and (45) for TM waves, then (43) and (46) takes the form

for TE

$$
\left(\frac{\partial^{2} H_{0 z}}{\partial x^{2}}\right)+\alpha\left(\frac{\partial^{2} H_{0 z}}{\partial y^{2}}\right)+\frac{\mu_{z z}}{\mu_{x x}}\left(k_{0}^{2} \alpha \varepsilon_{x x} \mu_{x x}-k^{2}\right) H_{0 z}=0
$$

for TM

$$
\left(\frac{\partial^{2} E_{0 z}}{\partial x^{2}}\right)+\alpha\left(\frac{\partial^{2} E_{0 z}}{\partial y^{2}}\right)+\frac{\varepsilon_{z z}}{\varepsilon_{x x}}\left(k_{0}^{2} \alpha \varepsilon_{x x} \mu_{x x}-k^{2}\right) E_{0 z}=0
$$

Incidentally, the case $\alpha \neq 1$ seems to be unrealistic. Therefore we will not consider it. In the second case, decoupling occurs if either

$$
\frac{\partial}{\partial y} \equiv 0 \text { or } \frac{\partial}{\partial x} \equiv 0
$$

which is fulfilled in an empty waveguide by the $\mathrm{TE}_{m 0}$ and $\mathrm{TE}_{0 n}$ modes respectively. These modes also exist in a waveguide filled with the biaxial medium. This was noticed for the first time in work of Damaskos et al. ${ }^{8}$ An Eq. (43) fulfilled by these solutions reduces to

$$
\begin{array}{ll}
\text { for } \mathrm{TE}_{m 0} & \frac{\partial^{2} H_{0 z}}{\partial x^{2}}+\frac{\mu_{z z}}{\mu_{x x}}\left(k_{0}^{2} \mu_{x x} \varepsilon_{y y}-k^{2}\right) H_{0 z}=0 \\
\text { for } \mathrm{TE}_{0 n} & \frac{\partial^{2} H_{0 z}}{\partial y^{2}}+\frac{\mu_{z z}}{\mu_{y y}}\left(k_{0}^{2} \varepsilon_{x x} \mu_{y y}-k^{2}\right) H_{0 z}=0
\end{array}
$$

while all other equations are satisfied identically. 


\section{APPENDIX B. WAVES IN A WAVEGUIDE}

\section{TE and TM waves in an uniaxially anisotropic medium}

Let's consider condition (47). In particular, it is satisfied for a medium with uniaxial anisotropy

$$
\varepsilon_{x x}=\varepsilon_{y y}, \quad \mu_{x x}=\mu_{y y}
$$

then equation (48) and (49) takes the form

$$
\frac{\partial^{2} \psi}{\partial x^{2}}+\frac{\partial^{2} \psi}{\partial y^{2}}+k_{c}^{2} \psi=0
$$

where

for TE

$$
\psi=H_{0 z}, \quad k_{c}^{2}=\frac{\mu_{z z}}{\mu_{x x}}\left(k_{0}^{2} \mu_{x x} \varepsilon_{x x}-k^{2}\right)
$$

for TM

$$
\psi=E_{0 z}, \quad k_{c}^{2}=\frac{\varepsilon_{z z}}{\varepsilon_{x x}}\left(k_{0}^{2} \mu_{x x} \varepsilon_{x x}-k^{2}\right)
$$

Boundary conditions on the waveguide inner surface $S$ can be expressed as

for TE

$$
\left.\left.(\hat{\mathbf{n}} \cdot \nabla \psi)\right|_{S} \triangleq \frac{\partial \psi}{\partial \hat{\mathbf{n}}}\right|_{S}=0
$$

for TM

$$
\left.\psi\right|_{S}=0
$$

where $\hat{\mathbf{n}}$ is unit normal vector at the surface.

Let's apply the separation of variables method. Writing $\psi$ in a typical way ${ }^{10}$

$$
\psi(x, y)=X(x) \cdot Y(y)
$$

and substituting into (53), we obtain formula

$$
\frac{\frac{\partial^{2} X(x)}{\partial x^{2}}}{X(x)}+\frac{\frac{\partial^{2} Y(y)}{\partial y^{2}}}{Y(y)}+k_{c}^{2}=0
$$

which leads to two equations of one variable

$$
\begin{aligned}
& \frac{\mathrm{d}^{2} X(x)}{\mathrm{d} x^{2}}+k_{x}^{2} X(x)=0 \\
& \frac{\mathrm{d}^{2} Y(y)}{\mathrm{d} y^{2}}+k_{y}^{2} Y(y)=0
\end{aligned}
$$

where $k_{x}$ and $k_{y}$ are separation constants. In addition, we obtain a relationship

$$
k_{c}^{2}=k_{x}^{2}+k_{y}^{2}
$$

The general solutions of (59) and (60) are respectively

$$
\begin{aligned}
& X(x)=A \sin \left(k_{x} x\right)+B \cos \left(k_{x} x\right) \\
& Y(y)=C \sin \left(k_{y} y\right)+D \cos \left(k_{y} y\right)
\end{aligned}
$$


Substituting the separated form of the solution (58) to the boundary conditions (57) and (56) one can obtain ${ }^{10}$ :

for TE

$$
\left.\frac{\mathrm{d} X(x)}{\mathrm{d} x}\right|_{x=0}=0,\left.\quad \frac{\mathrm{d} X(x)}{\mathrm{d} x}\right|_{x=a}=0,\left.\quad \frac{\mathrm{d} Y(y)}{\mathrm{d} y}\right|_{y=0}=0,\left.\quad \frac{\mathrm{d} Y(y)}{\mathrm{d} y}\right|_{y=b}=0
$$

for TM

$$
X(0)=0, \quad X(a)=0, \quad Y(0)=0, \quad Y(b)=0
$$

The general solution (62) differs from (63) by a spatial variable and by constant symbols only. We can substitute one of them to the appropriate boundary conditions and obtain the solution. Next, replacing the variable and symbols we can obtain the solution of the latter.

Substituting the general solution (62) to the boundary conditions, we obtain a system of equations

$$
\left\{\begin{array}{l}
A k_{x} \cos k_{x} 0-B k_{x} \sin k_{x} 0=0 \\
A k_{x} \cos k_{x} a-B k_{x} \sin k_{x} a=0
\end{array}\right.
$$

It gives $A=0$ and

$$
k_{x}=\frac{m \pi}{a}, \quad m=0,1,2, \ldots
$$

In the same way for the function $Y(y)$ we get $C=0$ and

$$
k_{y}=\frac{n \pi}{b}, \quad n=0,1,2, \ldots
$$

The product of solutions $X$ and $Y$ - function $\psi=H_{0 z}$ - is equal to

$$
H_{0 z}(x, y)=H_{0} \cos \left(\frac{m \pi}{a} x\right) \cos \left(\frac{n \pi}{b} y\right)
$$

where $H_{0}=B D$. This solution is called the $\mathrm{TE}_{m n}$ mode ${ }^{10}$. To avoid a trivial solution, at least one of the indices must be nonzero. The first index is conventionally associated with the larger dimension, so we assume $a \geq b$.

Another system of equations is obtained by substituting (62) to (65)

$$
\left\{\begin{array}{l}
A \sin \left(k_{x} 0\right)+B \cos \left(k_{x} 0\right)=0 \\
A \sin \left(k_{x} a\right)+B \cos \left(k_{x} a\right)=0
\end{array}\right.
$$

Hence $B=0$ and

$$
k_{x}=\frac{m \pi}{a}, \quad m=1,2,3, \ldots
$$

In turn, substituting (63) into (65) we have $D=0$ and

$$
k_{y}=\frac{n \pi}{b}, \quad n=1,2,3, \ldots
$$

The resulting solution $\psi=E_{0 z}$ is called $\mathrm{TM}_{m n}$ mode

$$
E_{0 z}(x, y)=E_{0} \sin \left(\frac{m \pi}{a} x\right) \sin \left(\frac{n \pi}{b} y\right)
$$

where $E_{0}=A C$. The separation constants are the same for both types of modes

$$
k_{x}=\frac{m \pi}{a}, \quad k_{y}=\frac{n \pi}{b}
$$


Substituting (72) to (61) we obtain the cutoff wave number $\left(k_{c}\right)_{m n}$ for the $n m$-th mode. It is designed $k_{m n}$, given by

$$
\left(k_{c}\right)_{m n} \equiv k_{m n}=\pi \sqrt{\left(\frac{m}{a}\right)^{2}+\left(\frac{n}{b}\right)^{2}}
$$

It is a function of the guide dimensions only. Taking into account (54) and (55), the wave number $k$ can be written as

for $\mathrm{TE}_{m n}$

$$
k=k_{0} \sqrt{\mu_{x x} \varepsilon_{x x}} \sqrt{1-\frac{1}{\varepsilon_{x x} \mu_{z z}} \frac{k_{m n}^{2}}{k_{0}^{2}}}
$$

for $\mathrm{TM}_{m n}$

$$
k=k_{0} \sqrt{\mu_{x x} \varepsilon_{x x}} \sqrt{1-\frac{1}{\mu_{x x} \varepsilon_{z z}} \frac{k_{m n}^{2}}{k_{0}^{2}}}
$$

The dispersion occurs, i.e. the relationship between the wave number $k$ and the angular frequency $\omega$ is nonlinear.

According to (36)-(40) transverse components for TE waves can be written as

$$
\begin{gathered}
E_{0 x}=-\frac{\mathrm{j} k}{k_{0}^{2} \mu_{x x} \varepsilon_{x x}-k^{2}}\left(\frac{\omega \mu_{0} \mu_{x x}}{k} \frac{\partial H_{0 z}}{\partial y}\right)=-\mathrm{j} \frac{\omega \mu_{0} \mu_{z z}}{k_{m n}^{2}} \frac{\partial H_{0 z}}{\partial y}=\mathrm{j} \frac{\omega \mu_{0} \mu_{z z}}{k_{m n}^{2}} \frac{n \pi}{b} H_{0} \cos \left(\frac{m \pi}{a} x\right) \sin \left(\frac{n \pi}{b} y\right) \\
E_{0 y}=-\frac{\mathrm{j} k}{k_{0}^{2} \mu_{x x} \varepsilon_{x x}-k^{2}}\left(-\frac{\omega \mu_{0} \mu_{x x}}{k} \frac{\partial H_{0 z}}{\partial x}\right)=\mathrm{j} \frac{\omega \mu_{0} \mu_{z z}}{k_{m n}^{2}} \frac{\partial H_{0 z}}{\partial x}=-\mathrm{j} \frac{\omega \mu_{0} \mu_{z z}}{k_{m n}^{2}} \frac{m \pi}{a} H_{0} \sin \left(\frac{m \pi}{a} x\right) \cos \left(\frac{n \pi}{b} y\right) \\
H_{0 x}=\mp \frac{1}{Z_{\mathrm{TE}}} E_{0 y}= \pm \mathrm{j} \frac{k \mu_{z z}}{k_{m n}^{2} \mu_{x x}} \frac{m \pi}{a} H_{0} \sin \left(\frac{m \pi}{a} x\right) \cos \left(\frac{n \pi}{b} y\right) \\
H_{0 y}= \pm \frac{1}{Z_{\mathrm{TE}}} E_{0 x}= \pm \mathrm{j} \frac{k \mu_{z z}}{k_{m n}^{2} \mu_{x x}} \frac{n \pi}{b} H_{0} \cos \left(\frac{m \pi}{a} x\right) \sin \left(\frac{n \pi}{b} y\right)
\end{gathered}
$$

where

$$
Z_{\mathrm{TE}}=\frac{\omega \mu_{0} \mu_{x x}}{k}
$$

Transverse components for TM waves can also be obtained from the same equations, hence

$$
\begin{gathered}
E_{0 x}=-\frac{\mathrm{j} k}{k_{0}^{2} \mu_{x x} \varepsilon_{x x}-k^{2}}\left( \pm \frac{\partial E_{0 z}}{\partial x}\right)=\mp \mathrm{j} \frac{k \varepsilon_{z z}}{k_{m n}^{2} \varepsilon_{x x}} \frac{\partial E_{0 z}}{\partial x}=\mp \mathrm{j} \frac{k \varepsilon_{z z}}{k_{m n}^{2} \varepsilon_{x x}} \frac{m \pi}{a} E_{0} \cos \left(\frac{m \pi}{a} x\right) \sin \left(\frac{n \pi}{b} y\right) \\
E_{0 y}=-\frac{\mathrm{j} k}{k_{0}^{2} \mu_{x x} \varepsilon_{x x}-k^{2}}\left( \pm \frac{\partial E_{0 z}}{\partial y}\right)=\mp \mathrm{j} \frac{k \varepsilon_{z z}}{k_{m n}^{2} \varepsilon_{x x}} \frac{\partial E_{0 z}}{\partial y}=\mp \mathrm{j} \frac{k \varepsilon_{z z}}{k_{m n}^{2} \varepsilon_{x x}} \frac{n \pi}{b} E_{0} \sin \left(\frac{m \pi}{a} x\right) \cos \left(\frac{n \pi}{b} y\right) \\
H_{0 x}=\mp \frac{1}{Z_{\mathrm{TM}}} E_{0 y}=\mathrm{j} \frac{\omega \varepsilon_{0} \varepsilon_{z z}}{k_{m n}^{2} \varepsilon_{x x}} \frac{n \pi}{b} E_{0} \sin \left(\frac{m \pi}{a} x\right) \cos \left(\frac{n \pi}{b} y\right) \\
H_{0 y}= \pm \frac{1}{Z_{\mathrm{TM}}} E_{0 x}=-\mathrm{j} \frac{\omega \varepsilon_{0} \varepsilon_{z z}}{k_{m n}^{2} \varepsilon_{x x}} \frac{m \pi}{a} E_{0} \cos \left(\frac{m \pi}{a} x\right) \sin \left(\frac{n \pi}{b} y\right)
\end{gathered}
$$

where

$$
Z_{\mathrm{TM}}=\frac{k}{\omega \varepsilon_{0} \varepsilon_{x x}}
$$


Note that

$$
\omega \mu_{0}=\omega \sqrt{\mu_{0} \varepsilon_{0}} \sqrt{\frac{\mu_{0}}{\varepsilon_{0}}}=k_{0} \eta, \quad \omega \varepsilon_{0}=\omega \sqrt{\mu_{0} \varepsilon_{0}} \sqrt{\frac{\varepsilon_{0}}{\mu_{0}}}=\frac{k_{0}}{\eta}
$$

where $\eta$ is the intrinsic impedance of the vacuum

$$
\eta=\sqrt{\frac{\mu_{0}}{\varepsilon_{0}}}
$$

Using these relationships, we get for TE waves

$$
\begin{gathered}
Z_{\mathrm{TE}}=\frac{\omega \mu_{0} \mu_{x x}}{k}=\frac{k_{0} \eta \mu_{x x}}{k}=\eta \frac{k_{0} \mu_{x x}}{k_{0} \sqrt{\mu_{x x} \varepsilon_{x x}} \sqrt{1-\frac{1}{\varepsilon_{x x} \mu_{z z}} \frac{k_{m n}^{2}}{k_{0}^{2}}}} \\
Z_{\mathrm{TE}}=\eta \frac{\sqrt{\frac{\mu_{x x}}{\varepsilon_{x x}}}}{\sqrt{1-\frac{1}{\varepsilon_{x x} \mu_{z z}} \frac{k_{m n}^{2}}{k_{0}^{2}}}}
\end{gathered}
$$

Similarly for TM waves we have

$$
\begin{gathered}
Z_{\mathrm{TM}}=\frac{k}{\omega \varepsilon_{0} \varepsilon_{x x}}=\eta \frac{k}{k_{0} \varepsilon_{x x}}=\eta \frac{k_{0} \sqrt{\mu_{x x} \varepsilon_{x x}} \sqrt{1-\frac{1}{\mu_{x x} \varepsilon_{z z}} \frac{k_{m n}^{2}}{k_{0}^{2}}}}{k_{0} \varepsilon_{x x}} \\
Z_{\mathrm{TM}}=\eta \sqrt{\frac{\mu_{x x}}{\varepsilon_{x x}}} \sqrt{1-\frac{1}{\mu_{x x} \varepsilon_{z z}} \frac{k_{m n}^{2}}{k_{0}^{2}}}
\end{gathered}
$$

\section{TE waves in a biaxially anisotropic medium}

Let's consider propagation of EM waves in the bianisotropic medium now. As mentioned in the previous chapter, the condition (50) resets TM modes. If $\partial / \partial y \equiv 0$ the TE modes fulfilled the relations

$$
\begin{gathered}
\frac{\partial^{2} H_{0 z}}{\partial x^{2}}+k_{c}^{2} H_{0 z}=0, \quad k_{c}^{2}=\frac{\mu_{z z}}{\mu_{x x}}\left(k_{0}^{2} \mu_{x x} \varepsilon_{y y}-k^{2}\right) \\
E_{0 y}=\frac{\mathrm{j}}{k_{0}^{2} \mu_{x x} \varepsilon_{y y}-k^{2}}\left(\omega \mu_{0} \mu_{x x} \frac{\partial H_{0 z}}{\partial x}\right)=\mathrm{j} \frac{\omega \mu_{0} \mu_{z z}}{k_{c}^{2}} \frac{\partial H_{0 z}}{\partial x} \\
H_{0 x}=\mp \frac{E_{0 y}}{Z_{m 0}}, \quad Z_{m 0}=\frac{\omega \mu_{0} \mu_{x x}}{k}
\end{gathered}
$$


Under condition $\partial / \partial x \equiv 0$ the other TE modes exist for which

$$
\begin{gathered}
\frac{\partial^{2} H_{0 z}}{\partial y^{2}}+k_{c}^{2} H_{0 z}=0, \quad k_{c}^{2}=\frac{\mu_{z z}}{\mu_{y y}}\left(k_{0}^{2} \varepsilon_{x x} \mu_{y y}-k^{2}\right) \\
E_{0 x}=\frac{\mathrm{j}}{k_{0}^{2} \varepsilon_{x x} \mu_{y y}-k^{2}}\left(-\omega \mu_{0} \mu_{y y} \frac{\partial H_{0 z}}{\partial y}\right)=-\mathrm{j} \frac{\omega \mu_{0} \mu_{z z}}{k_{c}^{2}} \frac{\partial H_{0 z}}{\partial y} \\
H_{0 y}= \pm \frac{E_{0 x}}{Z_{0 n}}, \quad Z_{0 n}=\frac{\omega \mu_{0} \mu_{y y}}{k}
\end{gathered}
$$

It can be shown, that solutions of (76) fulfilled boundary conditions (65) are $\mathrm{TE}_{m 0}$ modes ${ }^{8,9}$

$$
\begin{gathered}
H_{0 z}(x, y)=H_{0} \cos \left(\frac{m \pi}{a} x\right) \\
E_{0 y}=-\mathrm{j} \frac{\omega \mu_{0} \mu_{z z} a}{m \pi} H_{0} \sin \left(\frac{m \pi}{a} x\right) \\
H_{0 x}= \pm \mathrm{j} \frac{k \mu_{z z} a}{\mu_{x x} m \pi} H_{0} \sin \left(\frac{m \pi}{a} x\right) \\
E_{0 x}=E_{0 z}=H_{0 y}=0
\end{gathered}
$$

where

$$
k=k_{0} \sqrt{\mu_{x x} \varepsilon_{y y}} \sqrt{1-\frac{1}{\varepsilon_{y y} \mu_{z z}} \frac{k_{m 0}^{2}}{k_{0}^{2}}}, \quad\left(k_{c}\right)_{m 0} \equiv k_{m 0}=\frac{m \pi}{a}
$$

In turn, the solution of the (77) with boundary conditions (65) are $\mathrm{TE}_{0 n}$ modes

$$
\begin{gathered}
H_{0 z}(x, y)=H_{0} \cos \left(\frac{n \pi}{b} x\right) \\
E_{0 x}=\mathrm{j} \frac{\omega \mu_{0} \mu_{z z} b}{n \pi} H_{0} \sin \left(\frac{n \pi}{b} x\right) \\
H_{0 y}= \pm \mathrm{j} \frac{k \mu_{z z} b}{\mu_{y y} n \pi} H_{0} \sin \left(\frac{n \pi}{b} x\right) \\
E_{0 y}=E_{0 z}=H_{0 x}=0
\end{gathered}
$$

where

$$
k=k_{0}^{2} \sqrt{\varepsilon_{x x} \mu_{y y}} \sqrt{1-\frac{1}{\varepsilon_{x x} \mu_{z z}} \frac{k_{0 n}^{2}}{k_{0}^{2}}}, \quad\left(k_{c}\right)_{0 n} \equiv k_{0 n}=\frac{n \pi}{b}
$$




\section{APPENDIX C. DERIVATION OF THE MATRIX REPRESENTATION}

This appendix shows the derivation of the equation (14). The system of equations (12)-(13) can be written in the matrix form

$$
\frac{\mu_{z z}^{(l)}}{\mu_{z z}^{(l+1)}}\left[\begin{array}{cc}
\exp \left(-\mathrm{j} k^{(l)} d_{l}\right) & \exp \left(\mathrm{j} k^{(l)} d_{l}\right) \\
\frac{Z_{\mathrm{TE}}^{(l+1)}}{Z_{\mathrm{TE}}^{(l+1)}} \exp \left(-\mathrm{j} k^{(l)} d_{l}\right) & -\frac{Z_{\mathrm{TE}}^{(l+1)}}{Z_{\mathrm{TE}}^{(l+1)}} \exp \left(\mathrm{j} k^{(l)} d_{l}\right)
\end{array}\right]\left[\begin{array}{c}
A^{(l)} \\
B^{(l)}
\end{array}\right]=\left[\begin{array}{cc}
\exp \left(-\mathrm{j} k^{(l+1)} d_{l}\right) & \exp \left(\mathrm{j} k^{(l+1)} d_{l}\right) \\
\exp \left(-\mathrm{j} k^{(l+1)} d_{l}\right) & -\exp \left(\mathrm{j} k^{(l+1)} d_{l}\right)
\end{array}\right]\left[\begin{array}{l}
A^{(l+1)} \\
B^{(l+1)}
\end{array}\right]
$$

Introducing the wave impedances ratio

$$
Z^{(l, l+1)}=\frac{Z_{\mathrm{TE}}^{(l+1)}}{Z_{\mathrm{TE}}^{(l)}}
$$

we get

$$
\frac{\mu_{z z}^{(l)}}{\mu_{z z}^{(l+1)}}\left[\begin{array}{cc}
1 & 1 \\
Z^{(l, l+1)} & -Z^{(l, l+1)}
\end{array}\right]\left[\begin{array}{cc}
\exp \left(-\mathrm{j} k^{(l)} d_{l}\right) & 0 \\
0 & \exp \left(\mathrm{j} k^{(l)} d_{l}\right)
\end{array}\right]\left[\begin{array}{c}
A^{(l)} \\
B^{(l)}
\end{array}\right]=\left[\begin{array}{cc}
1 & 1 \\
1 & -1
\end{array}\right]\left[\begin{array}{cc}
\exp \left(-\mathrm{j} k^{(l+1)} d_{l}\right) & 0 \\
0 & \exp \left(\mathrm{j} k^{(l+1)} d_{l}\right)
\end{array}\right]\left[\begin{array}{l}
A^{(l+1)} \\
B^{(l+1)}
\end{array}\right]
$$

and next

$$
\left[\begin{array}{cc}
\mathrm{e}^{-\mathrm{j} k^{(l+1)} d_{l}} & 0 \\
0 & \mathrm{e}^{\mathrm{j} k^{(l+1)} d_{l}}
\end{array}\right]\left[\begin{array}{l}
A^{(l+1)} \\
B^{(l+1)}
\end{array}\right]=\frac{\mu_{z z}^{(l)}}{\mu_{z z}^{(l+1)}}\left[\begin{array}{cc}
1 & 1 \\
1 & -1
\end{array}\right]^{-1}\left[\begin{array}{cc}
1 & 1 \\
Z^{(l, l+1)} & -Z^{(l, l+1)}
\end{array}\right]\left[\begin{array}{cc}
\mathrm{e}^{-\mathrm{j} k^{(l)} d_{l}} & 0 \\
0 & \mathrm{e}^{\mathrm{j} k^{(l)} d_{l}}
\end{array}\right]\left[\begin{array}{l}
A^{(l)} \\
B^{(l)}
\end{array}\right]
$$

Let's define the matrix

$$
\mathbf{W}^{(l, l+1)}=\frac{\mu_{z z}^{(l)}}{\mu_{z z}^{(l+1)}}\left[\begin{array}{cc}
1 & 1 \\
1 & -1
\end{array}\right]^{-1}\left[\begin{array}{cc}
1 & 1 \\
Z^{(l, l+1)} & -Z^{(l, l+1)}
\end{array}\right]=\frac{\mu_{z z}^{(l)}}{\mu_{z z}^{(l+1)}} \frac{1}{2}\left[\begin{array}{cc}
1+Z^{(l, l+1)} & 1-Z^{(l, l+1)} \\
1-Z^{(l, l+1)} & 1+Z^{(l, l+1)}
\end{array}\right]
$$

After some manipulations, we get

$$
\left[\begin{array}{l}
A^{(l+1)} \\
B^{(l+1)}
\end{array}\right]=\left[\begin{array}{cc}
\mathrm{e}^{\mathrm{j} k^{(l+1)} d_{l}} & 0 \\
0 & \mathrm{e}^{-\mathrm{j} k^{(l+1)} d_{l}}
\end{array}\right] \mathbf{W}^{(l, l+1)}\left[\begin{array}{cc}
\mathrm{e}^{-\mathrm{j} k^{(l)} d_{l}} & 0 \\
0 & \mathrm{e}^{\mathrm{j} k^{(l)} d_{l}}
\end{array}\right]\left[\begin{array}{l}
A^{(l)} \\
B^{(l)}
\end{array}\right]
$$

As expected, the formula (78) takes the form (14).

\section{ACKNOWLEDGMENTS}

This research was funded by the Polish Ministry of Defence, grant number GBMON / 13-996 / 2018.

\section{REFERENCES}

[1] Markos, P. and Soukoulis, C. M., "Transmission properties and effective electromagnetic parameters of double negative metamaterials," Opt. Express 11(7), 649-661 (2003).

[2] Chen, X., Grzegorczyk, T. M., Wu, B., Pacheco, J. and Kong, J. A., "Robust method to retrieve the constitutive effective parameters of metamaterials," Phys. Rev. E., 70, 016608 (2004).

[3] Chen, H., Zhang, J., Bai, Y., Luo, Y., Ran, L., Jiang, Q., and Kong, J. A., "Experimental retrieval of the effective parameters of metamaterials based on a waveguide method," Opt. Express 14(26), 12944-12949 (2006).

[4] Castanié, A., Mercier, J.-F., Félix, S. and A. Maurel, "Generalized method for retrieving effective parameters of anisotropic metamaterials," Opt. Express 22(24), 29937-29953 (2014). 
[5] Akhtar, M.J., Feher, L.E. and Thumm, M., "A waveguide-based two-step approach for measuring complex permittivity tensor of uniaxial composite materials,", IEEE Trans. Microwave Theory Tech., 54(5), 2011-2022 (2006).

[6] Nicolson, A. M. and Ross, G. F., "Measurement of the intrinsic properties of materials by time-domain techniques," IEEE Trans. Instrum. Meas., IM-19, 377-382 (1970).

[7] W. B. Weir, "Automatic measurement of complex dielectric constant and permeability at microwave frequencies," Proc. of the IEEE, 62, 33-36 (1974).

[8] Damaskos, N.J., Mack, R. B., Maffett, A. L., Parmon, W. and Uslenghi, P. L. E., "The inverse problem for biaxial materials," IEEE Trans. Microwave Theory Tech., 32(4), 400-405 (1984).

[9] Uslenghi, P. L. E., "Corrections to "The inverse problem for biaxial materials"," IEEE Trans. Microwave Theory Tech., 40(1), 174 (1992).

[10] Griffiths, D. J., [Introduction to Electrodynamics], 3rd ed., PWN, Warszawa, 437-443 (2005), (Polish edition).

[11] Pozar, D. M, [Microwave Engineering], $4^{\text {th }}$ ed., John Wiley \& Sons, New York, 178-194 (2012).

[12] Balanis C. A., [Advanced engineering electromagnetics], John Wiley \& Sons, New York, 220-230 (1989).

[13] Born, M. and Wolf, E., [Principles of optics: electromagnetic theory of propagation, interference, and diffraction of light], 7th ed. Cambridge University Press, Cambridge, 54-70 (2005).

[14] Collin R. E., [Foundations for microwave engineering], 2nd ed., IEEE Press, New York, 248-260 (2001).

[15] Rulf B., "Transmission of microwaves through layered dielectrics - theory, experiment and application," Am. J. Phys., 56(1), 76-80 (1988).

[16] Xu, X., "Double waveguide method to retrieve the electromagnetic parameters of biaxial anisotropic materials," Electronics Letters, 54(21), 1224-1226 (2018).

[17] Ishizaki, T., Kida, S. and Awai, I., "A measurement method of material parameters for uniaxially anisotropic artificial dielectrics," IEICE Electronics Express, 7(11), 810-816 (2010).

[18] Kapelewski, J. and Dukata, A., "Electromagnetic wave behaviour in uniaxial magnetodielectric media of $1+1>2$ type," Acta Physica Polonica A, 116(3), 331-334 (2009). 